\title{
Noise insertion in CT for cocaine body packing: where is the limit of extensive dose reduction?
}

\author{
Joel Aissa ${ }^{1}$, Edwin Bölke $2^{*}$, Lino M. Sawicki ${ }^{1}$, Elisabeth Appel ${ }^{1}$, Christoph Thomas ${ }^{1}$, Philipp Heusch', \\ Martin Sedlmair ${ }^{3}$, Karl Krzymyk ${ }^{3}$, Patric Kröpil ${ }^{1,4}$, Gerald Antoch ${ }^{1}$ and Johannes Boos ${ }^{1}$
}

\begin{abstract}
Background: To evaluate the detection rate and image quality in CT-body-packer-screening at different radiationdose levels and to determine a dose threshold that enables a reliable detection of incorporated body packs and incidental findings with a maximum of dose saving.

Materials and methods: We retrospectively included 27 individuals who underwent an abdominal CT with automated exposure control due to suspected body packing. CT images were reconstructed at different radiation-dose levels of $50 \%, 10,5 \%$ and $1 \%$ using iterative reconstructions. All 135 CT reconstructions were evaluated by three independent readers. Reviewers determined the presence of foreign bodies and evaluated the image quality using a 5 -point ranking scale. In addition, visualization of incidental findings was assessed.

Results: A threshold of $5 \%$ (effective dose $0.11 \pm 0.07 \mathrm{mSv}$ ) was necessary to correctly identify all 27 patients with suspected body packing. Extensive noise insertion to a dose level of $1 \%(0.02 \pm 0.01 \mathrm{mSV})$ led to false-positive solid cocaine findings in three patients. Image quality was comparable between 100 and 50\%. The threshold for correct identification of incidental findings was 10\% of the initial dose (effective dose $0.21 \pm 0.13 \mathrm{mSv}$ ).

Conclusions: Our results indicate that dose of abdominal CT for the detection of intracorporeal cocaine body packets can be markedly reduced to up to $5 \%$ of the initial dose while still providing sufficient image quality to detect ingested body packets. However, a minimum effective dose of $0.21 \mathrm{mSv}$ ( $10 \%$ of initial dose) seems to be required to properly identify incidental findings.
\end{abstract}

Keywords: CT dose, Image analysis, Drug abuse, Low dose CT

\section{Introduction}

Concealment and transportation of cocaine is a growing business with worldwide impact and the transportation of drug containers by ingestion ("body packing") is a commonly used form of worldwide drug smuggling [1, 2]. Cocaine is not the only drug concealed by body packers, however; due to its price, cocaine is still the most frequently transported drug [3]. Leaky drug containers may lead to cocaine overdose, thus a fast and accurate

\footnotetext{
*Correspondence: Edwin.Boelke@med.uni-duesseldorf.de

2 Department of Radiation Oncology, Medical Faculty, University

Dusseldorf, Moorenstr. 5, 40225 Dusseldorf, Germany

Full list of author information is available at the end of the article
}

detection of body packets is required [4]. Abdominal plain radiography and CT localizer images are of limited value in the detection of body packets due to a low sensitivity $[5,6]$. Because of its high sensitivity, computed tomography $(\mathrm{CT})$ is the first line imaging modality in the detection of those drug couriers $[5,6]$. However, radiation exposure from a regular abdominal CT is considerably higher than from plain radiography and thus is a critical factor as body packers are usually young individuals $[3,7]$. Therefore, dose optimization is important in this special setting, and initial studies on dose optimization in body packing $\mathrm{CT}$ reported a dose reduction while retaining diagnostic image quality (IQ) $[5,8]$. However, the limit of extensive dose reduction in the setting of 
body packing was only investigated in experimental animal studies and cadavers but not in clinical studies with human individuals $[9,10]$.

The aim of our study was to evaluate the image quality, the diagnostic yield for body packets and the accuracy for the detection of incidental findings in suspected body packer abdominal CT to determine a dose threshold that enables reliable detection of incorporated body packets and incidental findings with a maximum of dose saving.

\section{Patient, materials and methods \\ Patient population}

This retrospective study was approved by the local ethics committee (IRB number: 5652). Twenty-seven individuals (20 male, 7 female) with a mean age of $37.8 \pm 11.1$ years (range 19-58 years) underwent a CT for suspected body packing between November 2014 and February 2016. All CTs were requested by the local customs authorities.

\section{Computed tomography protocol}

All CT examinations were performed on a 128-row dual source $\mathrm{CT}$ scanner using the single-source mode (Somatom Definition Flash, Siemens Healthineers, Erlangen, Germany). Scans were performed with a fixed tube voltage of $80 \mathrm{kVp}$ and automated tube current modulation (CarekV semi-mode, Siemens Healthineers) with a reference tube current time product of $60 \mathrm{mAs}$. The scan volume included the basal lungs to the proximal femur. Rotation time was $0.5 \mathrm{~s}$ and collimation was $0.6 \mathrm{~mm}$. Image quality of the CT protocol has been previously validated [11]. All scans were performed without oral or intra venous contrast media.

\section{Image reconstructions and processing}

Reconstructions were performed using an offline workstation and a prototype software device (ReconCT 13.8.2.0, Siemens Healthineers). Raw data is required to perform noise-insertion reconstructions. The system was calibrated prior to the reconstruction process by scanning a $20 \mathrm{~cm}$ water phantom with the same scan mode that is used for suspected body packer. The calibration data were transferred into the ReconCT software and defined as the standard for the noise simulation process. Reconstructions with simulated dose levels of $100 \%, 50 \%$, $10 \%, 5 \%$ and $1 \%$ of initial dose were performed using a validated noise-insertion tool (RawData Noise Insertion, ReconCT 13.8.2.0, Siemens Healthineers) [12]. All reconstructions were performed with iterative reconstruction (SAFIRE, Level 3) in axial orientation (3-mm slice thickness) using a medium smooth kernel (I30f) and a sharp kernel (I70f).

\section{Subjective image quality and pack identification}

All reconstructions were loaded onto an Advantage Windows Workstation (Fujitsu, Tokyo, Japan) and axial images were reviewed using a Digital Imaging and Communications in Medicine (DICOM) viewer software (VISI, 1.10.03, Siemens Healthineers). Evaluation was performed by three independent readers (xxx, yyy, and zzz) with 1,6 , and 8 years of experience in radiology, and all three readers were competent in the detection of solid and liquid body packs. The readers determined the presence and composition of foreign bodies (liquid or solid). The initial 100\% dose CT examination and the clinical $\mathrm{CT}$ reports served as reference standard. In cases positive on $\mathrm{CT}$, we received a stool analysis, which allowed confirmation of solid or liquid content. Readers evaluated the reduced dose reconstructions across all patients in a random order. Readers were blinded to the presence of body packets on the $100 \%$ reconstructions during the review. The $100 \%$ reconstructions were evaluated after review of the reduced dose reconstructions in a random order.

The confidence level for positive or negative body packing was evaluated using a 3 -point ranking scale $(1=$ low confidence in the diagnosis; $2=$ moderate confidence in the diagnosis; $3=$ excellent confidence in the diagnosis). The image quality was evaluated based on visualization of important structures as defined by the European Quality Criteria $[8,13]$ using a 5 -point ranking scale $(1=$ excellent image quality; $2=$ good image quality; $3=$ moderate image quality; $4=$ poor image quality; $5=$ nondiagnostic). Image quality was scored separately for the proper visualization of the liver parenchyma, the splenic parenchyma, the intestine, the perivascular retroperitoneal space, the pancreatic contours, the duodenum, the kidneys, the aorta, and the vena cava [13]. Mean attenuation of body packets $(\mathrm{HU})$ were analyzed by $3 \mathrm{ROI}$ measurements in all positive cases.

\section{Identification of secondary findings}

Secondary findings were defined as all findings that were not related to the body packing. Original reports were screened for secondary findings by aaa and reviewed in the $100 \%$ reconstructions (radiologist with 6 years of experience). There were twelve incidental findings in nine patients (Aortic vasosclerosis $n=3$; nephrolithiasis $n=1$; accessory spleen $n=2$; spondylolysis $n=1$; subcutaneous atheroma $n=1$; dysplasia of the hips $n=1$, liver cyst $n=1$, partial gastrectomy $n=1$; butterfly swirls $n=1$ ). Two radiologists (yyy and zzz) with 6 and 8 years of experience in radiology, who were blinded to the clinical data reviewed the $108 \mathrm{CT}$ reconstructions (dose levels $50 \%, 10 \%, 5 \%$, and $1 \%$ ) of the 27 patients for secondary 
findings in a random order. The $100 \%$ reconstructions were reviewed in a random order afterward.

Axial reconstructions with a smooth kernel (I30f) and a sharp kernel (I70f) were provided.

\section{Calculation of radiation dose}

Volumetric computed tomography dose index $\left(\mathrm{CTDI}_{\mathrm{vol}}\right)$ and Dose Length Product (DLP) were extracted from the Picture Archiving and Communication System (PACS). To estimate the effective radiation dose, DLP values were converted to Millisieverts ( $\mathrm{mSv}$ ) by using conversion factors provided by the American Association of Physicists in Medicine (AAPM) for abdominal CT examinations $(0.015 \mathrm{mSv} / \mathrm{mGycm})$ [14]. To estimate the potential dose saving, the effective dose at the simulated dose levels $(50 \%, 10 \%, 5 \%$, and $1 \%$ of initial effective dose) was calculated. Patient's constitution was evaluated by abdominal diameter measurements. Therefore, we analyzed the lateral (Dlat) and anterior to posterior diameter (Dap) at the level of largest diameter in abdominal axial slices. Measurements were performed by an independent reader (zzz) with 8 years of experience in radiology. The effective Diameter (Deff) was calculated using the obtained Dlat and Dap [15].

$$
\text { Deff }=\sqrt{(\text { Dlat } \times \text { Dap })}
$$

\section{Statistical analysis}

IBM SPSS Statistics 21 for Windows (IBM, SPSS Statistics 21, Chicago, IL) was used for statistical analysis. Values for subjective image quality are reported as media $n \pm$ interquartile range (IQR). A Kolmogorovtest was performed to test for normality. A Wilcoxon test was used as a nonparametric test for paired values. A Chi-square test was performed to compare the different groups. Kappa-value was calculated to evaluate the interobserver agreement. Interobserver agreement was defined as excellent $(\kappa>0.80)$, good $(\kappa=0.61-0.80)$, moderate $(\kappa=0.41-0.60)$, fair $(\kappa=0.21-0.40)$, and poor $(\mathrm{k} \leq 0.20)$ [16]. Level of statistical significance was set to $<0.05$.

\section{Results}

\section{Radiation dose}

$\mathrm{CTDI}_{\mathrm{vol}}$ and DLP of our standard institutional CT protocol for the detection of body packets were $2.96 \pm 1.83 \mathrm{mGy}$ (range 1.09-8.94 mGy) and 140.0 \pm 88.1 mGycm (range 49-393 mGycm). The effective dose was $2.10 \pm 1.32 \mathrm{mSv}$ (range 0.74-5.89 $\mathrm{mSv}$ ). The mean tube current was $150.7 \pm 93.5 \mathrm{mAs}$. The effective doses of the $50 \%, 10 \%, 5 \%$, and $1 \%$ reconstructions are shown in Table 1. The mean Dlat was $34.2 \pm 5.8 \mathrm{~cm}$ (range 48.4$27.6 \mathrm{~cm}$ ) and the mean Dap was $25.7 \pm 5.9 \mathrm{~cm}$ (range
Table 1 The overall estimated dose results (CTDI $_{\text {vol }}$ DLP, and effective dose), due to dose level at $100 \%$ and the estimated dose savings at $50 \%, 10,5 \%$, and $1 \%$ of initial dose

\begin{tabular}{lll}
\hline \multicolumn{1}{c}{$\begin{array}{c}\text { CTDI } \\
\text { mean } \pm \text { SD }\end{array}$} & $\begin{array}{l}\text { DLP }(\mathbf{m G y c m}) \\
\text { mean } \pm \text { SD }\end{array}$ & $\begin{array}{l}\text { Eff. dose } \\
(\mathbf{m S v}) \\
\text { mean } \pm \text { SD }\end{array}$ \\
\hline $\begin{array}{ll}\text { Full dose (\%) } \\
100 \quad 2.96 \pm 1.83\end{array}$ & $140 \pm 88.1$ & $2.10 \pm 1.32$ \\
Estimated dose savings (\%) & & $1.05 \pm 0.66$ \\
50 & & $0.21 \pm 0.13$ \\
10 & & $0.11 \pm 0.07$ \\
5 & & $0.02 \pm 0.01$ \\
\hline
\end{tabular}

$35.7-17.3 \mathrm{~cm}$ ). The mean Deff was $29.9 \pm 5.9 \mathrm{~cm}$ (range 22.4-39.1).

\section{Body pack identification}

At the dose level of $100 \%$ the three observers detected incorporated body packets in $8 / 27$ individuals (29.6\%). All patients with body packets hat swallowed multiple body packets ( $>30$ packets per patient). All readers correctly identified liquid $(1 / 8,12.5 \%)$ and solid $(7 / 8,87.5 \%)$ body packets.

All body packets were properly identified at a dose level of $50 \%, 10 \%$, and $5 \%$ (Fig. 1). However, one reader falsely classified one body packet as solid instead of liquid at a dose level of $5 \%$. We found an excellent interobserver agreement of all three readers for $100 \%, 50 \%$, and $10 \%$ $(\kappa=1)$. For $5 \%$, we found a $\kappa$ value of 0.91 between reader 1 and 2 . At $1 \%$ of the initial dose, false-positive findings led to a significantly higher number of detected body packer cases (body packing cases: 8/27, 29.6\%; reader 1 : $8 / 27,29.6 \%$, reader $2: 9 / 27,33.3 \%$, reader $3: 10 / 27,37.0 \%$; $\kappa$ reader 1 vs. $2=0.17, \kappa$ value reader 1 vs. $3=0.91, \kappa$ value reader 2 vs. $3=0.11 ; \mathrm{p}=0.03$ ) (Fig. 2 ). The mean density of body packets was $292.2 \pm 69.7 \mathrm{HU}$ (range 391.3-157.7).

Concerning the confidence level for positive or negative body packing, we found no differences between $100 \%$ and all other dose levels (dose level 100\%: median $3 \pm 2$; dose level $50 \%$ : median $3 \pm 1, \mathrm{p}=0.73$; dose level $10 \%$ : median $3 \pm 1, \mathrm{p}=0.85$; dose level $5 \%$ : median $3 \pm 1$, $\mathrm{p}=0.47$; dose level $1 \%: 3 \pm 1.5, \mathrm{p}=0.34$ ).

\section{Subjective image quality}

Subjective image quality was significantly reduced for all simulated dose levels compared to the reference standard (dose level 100\%: median $1 \pm 2$; dose level $50 \%$ : median $2 \pm 2.5 ; \mathrm{p}=0.023$; dose level $10 \%$ : median $3 \pm 2$, 


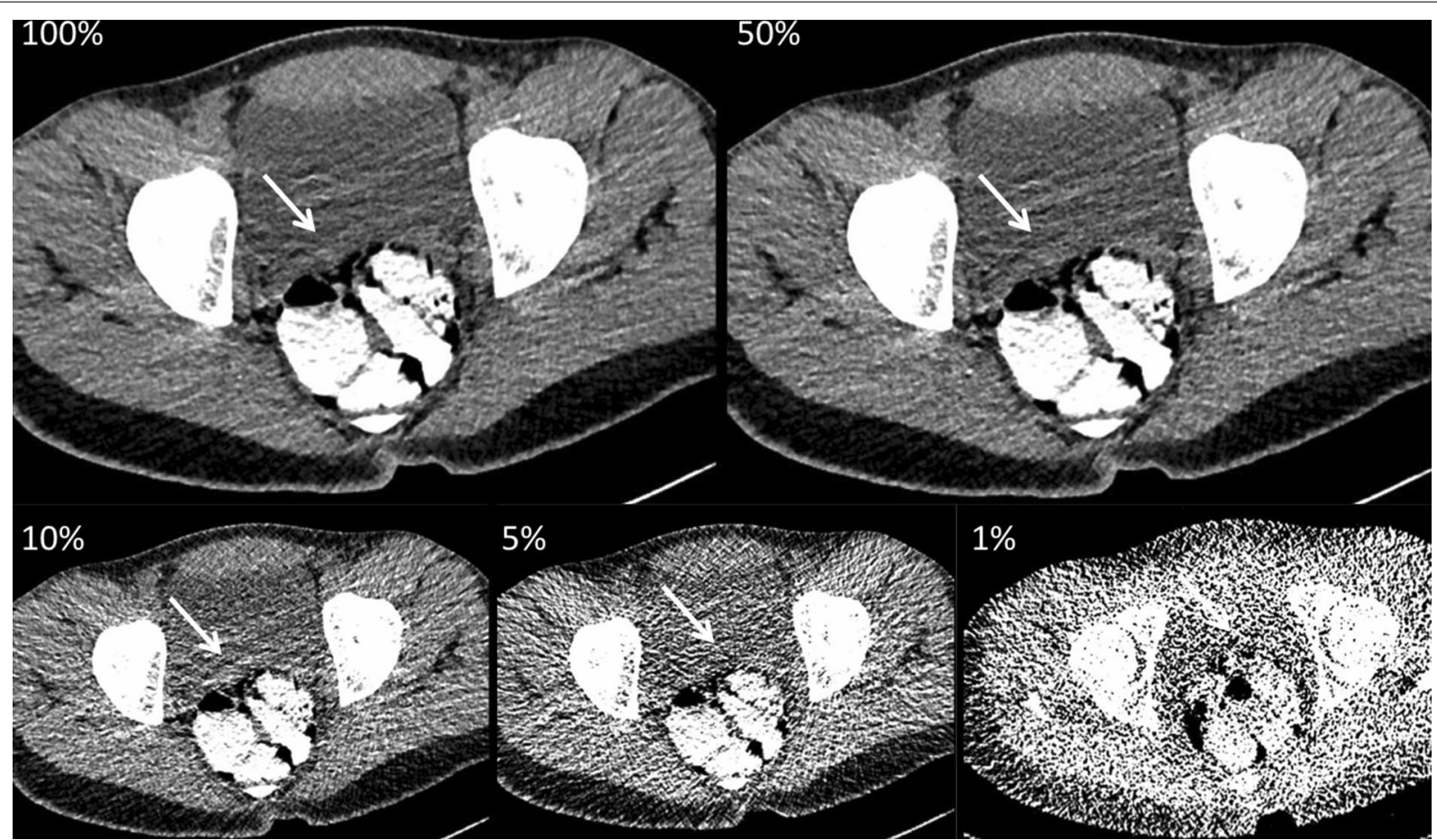

Fig. 1 A 22-year-old body packer, who was examined with $80 \mathrm{kVp}$ and automated tube current modulation (tube current time product: $88 \mathrm{mAs}$, effective dose at 100\%: $1.16 \mathrm{mSv}$ ). The axial reconstructions (soft tissue window, window level 40/300) with stepwise reduced dose levels (100\%, $50 \%, 10 \%, 5 \%$, and $1 \%$ ) show multiple liquid body packets (white arrows) which are hyperattenuating compared to the surrounding bowel content. While differentiation of bowel and that of urinary bladder decrease within stepwise dose reduction, liquid cocaine remains easily detectable with only $1 \%$ of initial radiation dose (effective dose: $0.01 \mathrm{mSv}$ )

$\mathrm{p}<0.0001$; dose level 5\%: median $4 \pm 2$, $\mathrm{p}<0.0001$; dose level $1 \%$ : median $5 \pm 2, \mathrm{p}<0.0001)$. At $100 \%$ and $50 \%$ of radiation dose, all CT scans were rated diagnostic while, at $10 \%, 5 \%$, and $1 \%$, all three readers rated several CT scans as nondiagnostic (10\%: $n=13 ; 5 \%: n=24 ; 1 \%$ : $n=42$ ).

\section{Identification of secondary findings}

At the radiation-dose levels of 100,50 , and $10 \%$, all incidental findings $(12 / 12,100 \%)$ were detected by both readers (Fig. 3). Detection of incidental findings was significantly worse at $5 \%(6 / 12,50 \%)$ and $1 \%(3 / 12,25 \%)$ ( $\mathrm{p}<0.001$ for both).

\section{Discussion}

We found abdominal CT for suspected body packer with an effective dose of $1.05 \mathrm{mSV}$ to enable reliable detection of body packets in our collective. Subjective image quality was reduced for all reduced dose levels compared to $100 \%$ of the initial radiation dose. In our collective, $10 \%$ of the initial dose $(0.21 \mathrm{mSV})$ was required to provide sufficient image quality for the detection of secondary findings.
Individuals in our study were of young age with a mean of $37.8 \pm 11.1$ years. This is in accordance to previous studies on CT body packing that found individuals suspected of body packing to be mainly young adults $[3,7]$. Dose saving in young adults is desirable, however; for reasons of possible body packer complications or legal consequences, the maintenance of a diagnostic image quality is mandatory. Noncontrast $\mathrm{CT}$ is superior to plain radiography in the detection of incorporated body packets and currently reflects the reference standard in the detection of ingested drug containers $[5,6]$. However, young age and the strict indication due to radiation exposure in otherwise healthy individuals require a focus on radiation-dose optimization. Magnetic resonance imaging (MRI) and Sonography are possible alternatives without radiation exposure. Due to this special setting with armed customer officers and frequently noncompliant delinquents, these methods pose complications during performance in clinical routine. However, MRI and Sonography seem to be suitable alternatives in cases of pregnancy and children [17].

We found an effective dose of $0.11 \mathrm{mSv}$ to be sufficient for the detection of cocaine body packets. Prior 


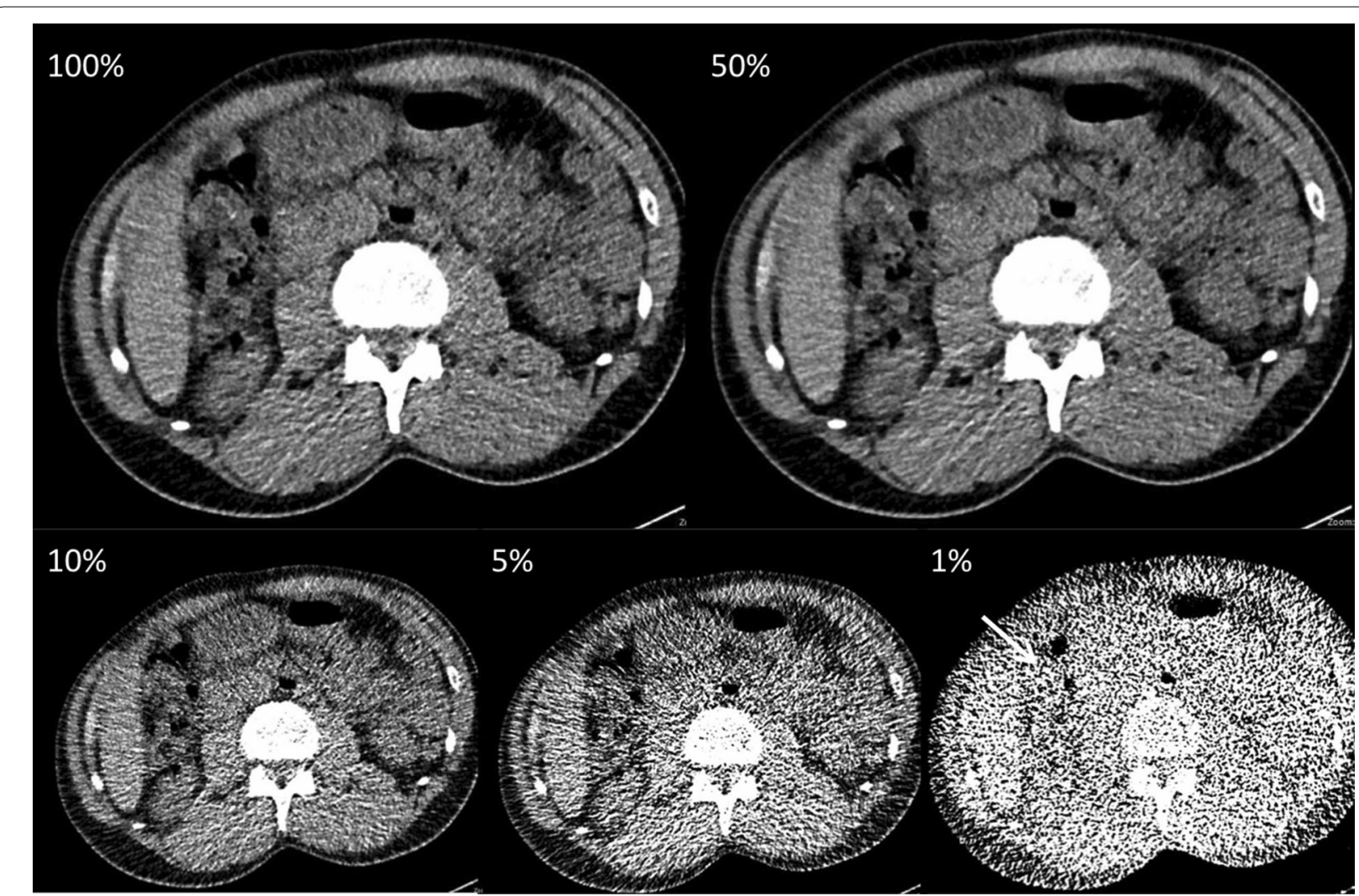

Fig. 2 A 58-year-old individual suspected of body packing. CT examination was performed at $80 \mathrm{kVp}$ with ATCM (tube current time product: 81 mAs, dose level 100\% effective dose at 100\%: 1.09 mSv). Axial CT scans (soft tissue window, window level 40/300) with stepwise dose reduction $(100 \%, 50 \%, 10 \%, 5 \%$, and $1 \%)$. In this case at $1 \%$ of initial dose $(0.02 \mathrm{mSv})$, one additional false-positive solid body packet was rated by reader one (white arrow)

studies evaluated body packing CT protocols with reduced radiation dose that are used in clinical routine and reported diagnostic image quality for $\mathrm{CT}$ protocols with an effective dose of 1.06-2.05 $\mathrm{mSv}[5,8,11,18,19]$ which is close to an abdominal radiography. However, due to the clinical setting of previous studies, a radiation-dose threshold could not be determined.

To our knowledge, extensive dose reduction in the context of body packing was investigated only by Maurer et al. [9] in an animal model and Laberke et al. in a postmortem study [10]. Maurer et al. [9] investigated a total number of twelve solid cocaine containers, which were introduced into the intestine of crossbred pigs. The pigs underwent repeated CT examinations with a fixed tube voltage of $80 \mathrm{kVp}$ and a stepwise reduction of tube current from 350 to $10 \mathrm{mAs}$. The threshold for correct detection of all containers was $125 \mathrm{mAs}$, which has resulted in an effective dose of $1.0 \mathrm{mSv}$. This is ten times higher than the $0.1 \mathrm{mSv}$ found in our study in humans. Laberke et al. placed up to 20 body packets in the alimentary tract of human cadavers. They showed a threshold of $0.6 \mathrm{mSv}$ for the correct identification of body packets. Due to different postmortem changes in animal and human cadavers, the image interpretation of CT scans can differ compared to living individuals. Therefore, our study is the first study which evaluated an extensive dose reduction in the context of body packing in clinical routine.

We found a mean attenuation of body packets of 292.2 HU. The mean attenuation of body packs in the study by Maurer et al. [9] was $-69.6 \mathrm{HU}$ (range 135-247 HU). The negative values seem to be the reason for the high threshold of $125 \mathrm{mAs}$ for correct identification in the animal study. We did not find any cocaine containers with negative density values and cannot give an explanation for the negative values reported by Maurer et al. [9]. Negative values usually are attributable to the admixture of the content, which is usually not a pure form of cocaine. However, most studies that investigated cocaine body packing on CT reported density values comparable to our results [5, 6, 8, 19-21].

Of note, the noise-insertion tool used herein has been previously validated and allows for a reliable simulation of radiation-dose reduction in CT [12]. This allows for 


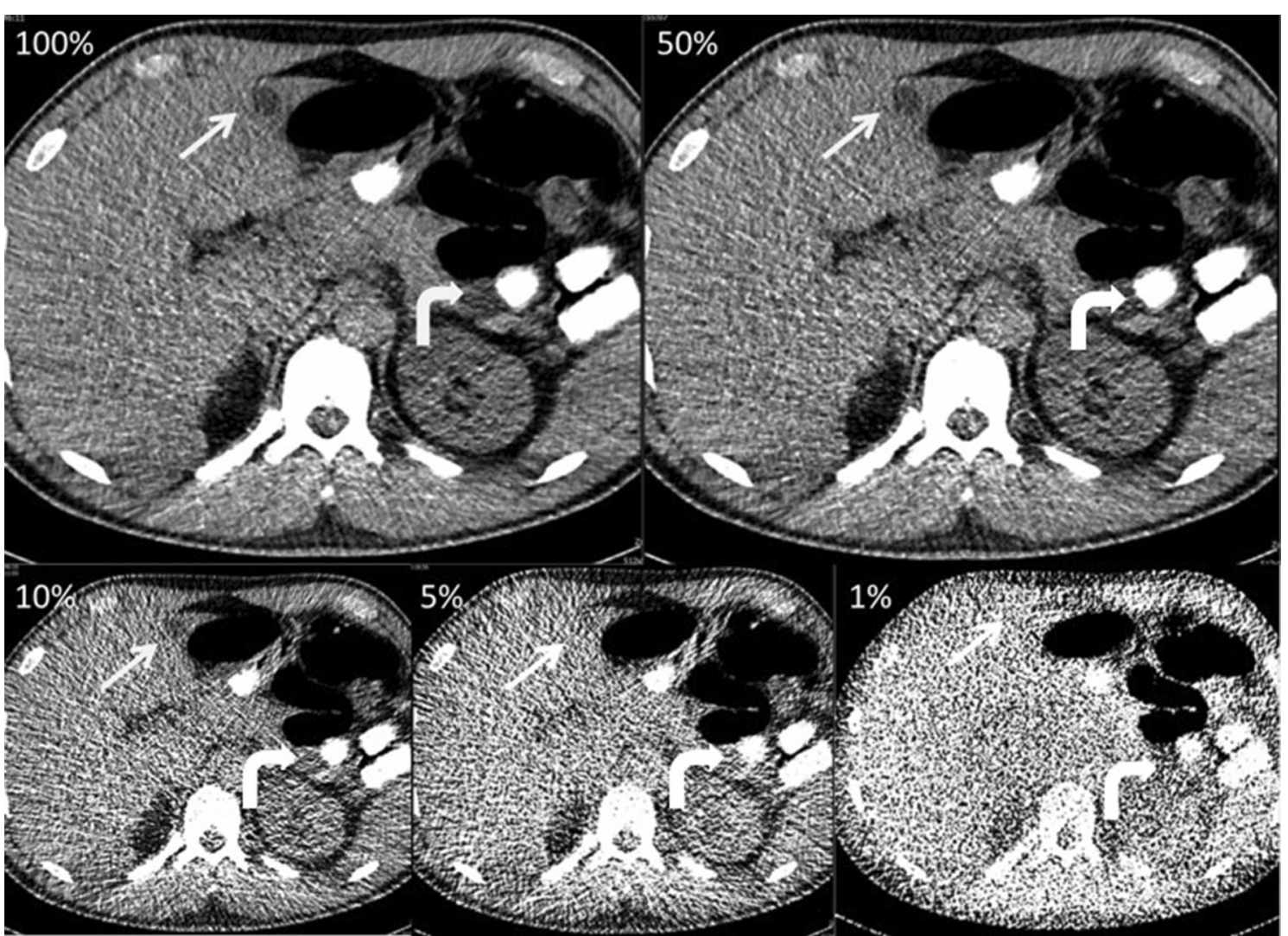

Fig. 3 A 40-year-old body packer, who was examined with $80 \mathrm{kVp}$ and automated tube current modulation (tube current time product: 134 mAs, effective dose at 100\%: $1.97 \mathrm{mSv}$ ). The axial reconstructions (soft tissue window, window level 40/300) with stepwise dose reduction (100\%, 50\%, $10 \%, 5 \%$, and $1 \%$ ) show multiple solid body packets (white curved arrows), which remained easily detectable at only $1 \%$ of initial dose (0.02 mSv). The liver cyst (white arrows) was missed by all readers at dose level $5 \%$ and $1 \%$

the assessment of radiation-dose thresholds in regards to diagnostic yield in a clinical setting while not exposing patients to additional radiation. Additional CT examinations for research purposes have been reported, and the ethical considerations were discussed [22, 23]. A recent study reported a technique to reconstruct multiple dose levels from a dual-energy CT examination [23]. However, even in this study, an additional CT examination with a not-clinically indicated radiation exposure for the patients was necessary. The noise simulation tool used in our study may help to improve research results for radiation-dose reduction without exposing patients to unnecessary radiation.

We found a significant decline of image quality upon decreasing the radiation dose. At $10 \%$ of the initial dose, several CT examinations were rated nondiagnostic based on the European Quality Criteria [13]. However, screening for body packing is a special task, and $10 \%$ of the initial dose provided sufficient image quality for detection of all body packets.
It was found in former studies that a Deff of $30 \mathrm{~cm}$ correlates with a BMI of 26 in adults undergoing an abdominal CT [15]. As the mean Deff was $29.9 \mathrm{~cm}$ in this study, the averaged physical constitution of the investigated individuals can be described as obese. Based on this, the findings of this study regarding relevant dose reduction by preserving diagnostic image quality gain even more significance.

Other techniques like the dual-energy CT have evaluated if heroin and cocaine can be distinguished using dual-energy CT. They showed that the slope of the spectral curve and the DEI from dual-energy CT data can be used to distinguish heroin and cocaine in vitro [24].

All incidental findings were detected with 100\%, 50\% and $10 \%$ of initial dose. At dose reduction level $5 \%$ and $1 \%$ detection of incidental findings was significantly worse. There is only one study which evaluated incidental findings detected by noncontrast CT scans in the setting of body packing [25]. Overall, they found 31 incidental findings in $18 \mathrm{CT}$ scans. There was no information 
concerning the performed CT scan protocol. We showed that incidental findings are reliably detectable with an effective dose of $0.21 \mathrm{mSv}$. The evaluated effective doses are specific to the manufacturer, hardware, and level of iterative reconstruction used in this study as explained in "Patient, materials and methods" section.

Our study has limitations. First, we performed a retrospective study, and the number of individuals with body packets was limited. Moreover, according to the small number of positive cases 'reader memory' effects on the detection of body packs and secondary effects were possible. In addition, a software tool was used to model dose reduction, however, the method has been previously validated. Due to the software limitations, we only reconstructed axial CT images. Second, we only investigated drug containers made from cocaine with a limited range of packet density; however, cocaine is the most frequently concealed drug transported by body packers [3]. Third, our study population did not include very obese individuals for whom our results might not be applicable. Last, stool analysis was only available in cases positive on CT. However, CT is regarded as the reference standard for the detection of body packets and thus we think that the reference standard applied in our study, which was also based on full-dose abdominal CT scans was adequate $[5,6,9]$. All body packing patients had ingested multiple packets. Results may differ for patients with a single packet; however, from our experience, this reflects clinical routine as body packers typically swallow multiple packets.

\section{Conclusion}

Our results indicate that dose of abdominal CT for the detection of intracorporeal cocaine body packets can be markedly reduced to up to $5 \%$ of the initial dose while still providing sufficient image quality to detect ingested body packets. However, an effective dose of $0.21 \mathrm{mSv}$ ( $10 \%$ of initial dose) seems to be required to properly identify secondary findings.

\section{Authors' contributions}

$\mathrm{BB}, \mathrm{HS}, \mathrm{LS}, \mathrm{EA}, \mathrm{PK}$, and GA carried out the studies, participated in collecting data, and drafted the manuscript. EB, KK, and MS performed the statistical analysis and participated in its design. EB, PK, JB, PH, and CT helped to draft the manuscript. All authors read and approved the final manuscript.

\section{Author details}

${ }^{1}$ Department of Diagnostic and Interventional Radiology, Medical Faculty, University Dusseldorf, Moorenstr. 5, 40225 Dusseldorf, Germany. ${ }^{2}$ Department of Radiation Oncology, Medical Faculty, University Dusseldorf, Moorenstr. 5, 40225 Dusseldorf, Germany. ${ }^{3}$ Computed Tomography, Siemens Healthineers GmbH, Forchheim, Germany. ${ }^{4}$ Department of Radiology, BG Klinikum Duisburg gGmbH, 47249 Duisburg, Germany.

\section{Competing interests}

The authors declare that they have no competing interests.
Availability of data and materials

All data and materials can be found in "Patient, materials and methods" section or can be accessed via JA and PK.

\section{Consent for publication}

All authors have given consent for publication.

Ethics approval and consent to participate

This study was approved by the local ethics committee.

\section{Funding}

There was no funding for this investigation.

\section{Publisher's Note}

Springer Nature remains neutral with regard to jurisdictional claims in published maps and institutional affiliations.

Received: 13 June 2018 Accepted: 29 November 2018

Published online: 07 December 2018

References

1. Traub SJ, Hoffman RS, Nelson LS. Body packing-the internal concealment of illicit drugs. N Engl J Med. 2003;349(26):2519-26.

2. Deitel M, Syed AK. Intestinal obstruction by an unusual foreign body. Can Med Assoc J. 1973;109(3):211-2.

3. Cappelletti S, Piacentino D, Sani G, et al. Systematic review of the toxicological and radiological features of body packing. Int J Leg Med. 2016;130(3):693-709.

4. Wetli CV, Mittlemann RE. The "body packer syndrome"-toxicity following ingestion of illicit drugs packaged for transportation. J Forensic Sci. 1981;26(3):492-500

5. Poletti PA, Canel L, Becker CD, et al. Screening of illegal intracorporeal containers ("body packing"): is abdominal radiography sufficiently accurate? A comparative study with low-dose CT. Radiology. 2012;265(3):772-9.

6. Bulakci M, Kalelioglu T, Bulakci BB, Kiris A. Comparison of diagnostic value of multidetector computed tomography and X-ray in the detection of body packing. Eur J Radiol. 2013. https://doi.org/10.1016/j.ejrad .2012.12.022.

7. Beno S, Calello D, Baluffi A, Henretig FM. Pediatric body packing: drug smuggling reaches a new low. Pediatr Emerg Care. 2005;21(11):744-6.

8. Aissa J, Rubbert C, Boos J, et al. Low-tube voltage $100 \mathrm{kVp}$ MDCT in screening of cocaine body packing: image quality and radiation dose compared to 120 kVp MDCT. Abdom Imaging. 2015. https://doi. org/10.1007/s00261-015-0464-2.

9. Maurer MH, Niehues SM, Schnapauff D, et al. Low-dose computed tomography to detect body-packing in an animal model. Eur J Radiol. 2011;78(2):302-6.

10. Laberke PJ, Blum S, Waelti S, et al. Systematic evaluation of radiation dose reduction in ct studies of body packers: accuracy down to submillisievert levels. AJR Am J Roentgenol. 2016;206(4):740-6. https://doi.org/10.2214/ AJR.15.15374.

11. Aissa J, Boos J, Rubbert C, et al. Optimizing radiation exposure in screening of body packing: image quality and diagnostic acceptability of an $80 \mathrm{kVp}$ protocol with automated tube current modulation. Forensic Sci Med Pathol. 2017;13(2):145-50. https://doi.org/10.1007/s1202 4-017-9848-7.

12. Kramer M, Ellmann S, Allmendinger T, et al. Computed tomography angiography of carotid arteries and vertebrobasilar system. Medicine (Baltimore). 2015. https://doi.org/10.1097/md.0000000000001058.

13. Båth M, Månsson LG. Visual grading characteristics (VGC) analysis: a nonparametric rank-invariant statistical method for image quality evaluation. Br J Radiol. 2007;80(951):169-76.

14. McCollough C, Cody D, Edyvean S, Geise R, Gould B, Keat N, Huda W, Judy P, Kalender W, McNitt-Gray M, Morin R. The measurement, reporting, and management of radiation dose in CT. Am Assoc Phys Med. 2008;23(23):1-28. 
15. Boos J, Kröpil P, Bethge OT, et al. Accuracy of size-specific dose estimate calculation from center slice in computed tomography. Radiat Prot Dosimetry. 2017. https://doi.org/10.1093/rpd/ncx119.

16. Landis JR, Koch GG. The measurement of observer agreement for categorical data. Biometrics. 1977;33(1):159-74.

17. Sammet S. Magnetic resonance safety. Abdom Radiol NY. 2016:41(3):444-51. https://doi.org/10.1007/s00261-016-0680-4.

18. Pache G, Einhaus D, Bulla S, Baumann T, Langer M, Blanke P. Low-dose computed tomography for the detection of cocaine body packs: clinical evaluation and legal issues. Rofo. 2012;184(2):122-9.

19. Aissa J, Kohlmeier A, Rubbert C, et al. Diagnostic value of CT-localizer and axial low-dose computed tomography for the detection of drug body packing. J Forensic Leg Med. 2016;37:55-60. https://doi.org/10.1016/j. jflm.2015.10.009.

20. Flach PM, Ross SG, Ampanozi G, et al. "Drug mules" as a radiological challenge: sensitivity and specificity in identifying internal cocaine in body packers, body pushers and body stuffers by computed tomography, plain radiography and Lodox. Eur J Radiol. 2012;81(10):2518-26. https://doi. org/10.1016/j.ejrad.2011.11.025.
21. Schmidt S, Hugli O, Rizzo E, et al. Detection of ingested cocainefilled packets-diagnostic value of unenhanced CT. Eur J Radiol. 2008:67(1):133-8.

22. Yin W-H, Lu B, Li N, et al. Iterative reconstruction to preserve image quality and diagnostic accuracy at reduced radiation dose in coronary CT angiography: an intraindividual comparison. JACC Cardiovasc Imaging. 2013;6(12):1239-49. https://doi.org/10.1016/j.jcmg.2013.08.008.

23. Bellini D, Ramirez-Giraldo JC, Bibbey A, et al. Dual-source single-energy multidetector $\mathrm{CT}$ used to obtain multiple radiation exposure levels within the same patient: phantom development and clinical validation. Radiology. 2016. https://doi.org/10.1148/radiol.2016161233.

24. Grimm J, Wudy R, Ziegeler E, et al. Differentiation of heroin and cocaine using dual-energy CT-an experimental study. Int J Legal Med. 2014;128(3):475-82. https://doi.org/10.1007/s00414-014-0980-6.

25. Médiouni Z, Steffen M, Bécour B. Incidentalomas revealed by abdominopelvic computed tomography scans performed in body packers. Am J Forensic Med Pathol. 2013;34(4):331-4. https://doi.org/10.1097/PAF.0b013 e31829f6901.
Ready to submit your research? Choose BMC and benefit from:

- fast, convenient online submission

- thorough peer review by experienced researchers in your field

- rapid publication on acceptance

- support for research data, including large and complex data types

- gold Open Access which fosters wider collaboration and increased citations

- maximum visibility for your research: over $100 \mathrm{M}$ website views per year

At BMC, research is always in progress.

Learn more biomedcentral.com/submissions 